\title{
Individual Differences in Social Behavior and Cortical Vasopressin Receptor: Genetics, Epigenetics, and Evolution
}

\author{
Steven M. Phelps ${ }^{1 *}$, Mariam Okhovat ${ }^{1,2}$ and Alejandro Berrio ${ }^{1,3}$ \\ 1 Department of Integrative Biology, University of Texas at Austin, Austin, TX, United States, ${ }^{2}$ Department of Medicine, \\ Oregon Health and Science University, Portland, OR, United States, ${ }^{3}$ Department of Biology, Duke University, Durham, NC, \\ United States
}

OPEN ACCESS

Edited by:

Aras Petrulis,

Georgia State University,

United States

Reviewed by:

Alexa H. Veenema,

Michigan State University,

United States

Lisa A. McGraw,

North Carolina State University,

United States

${ }^{*}$ Correspondence:

Steven M. Phelps

sphelps@mail.utexas.edu

Specialty section: This article was submitted to Neuroendocrine Science, a section of the journal

Frontiers in Neuroscience

Received: 05 July 2017 Accepted: 14 September 2017

Published: 04 October 2017

Citation:

Phelps SM, Okhovat M and Berrio A (2017) Individual Differences in Social

Behavior and Cortical Vasopressin Receptor: Genetics, Epigenetics, and

Evolution. Front. Neurosci. 11:537.

doi: 10.3389/fnins.2017.00537
Social behavior is among the most complex and variable of traits. Despite its diversity, we know little about how genetic and developmental factors interact to shape natural variation in social behavior. This review surveys recent work on individual differences in the expression of the vasopressin 1 a receptor (V1aR), a major regulator of social behavior, in the neocortex of the socially monogamous prairie vole. $\mathrm{V} 1 \mathrm{aR}$ exhibits profound variation in the retrosplenial cortex (RSC), a region critical to spatial and contextual memory. RSC-V1aR abundance is associated with patterns of male space-use and sexual fidelity in the field: males with high RSC-V1aR show high spatial and sexual fidelity to partners, while low RSC-V1aR males are significantly more likely to mate outside the pair-bond. Individual differences in RSC-V1aR are predicted by a set of linked single nucleotide polymorphisms within the avpr1a locus. These alternative alleles have been actively maintained by selection, suggesting that the brain differences represent a balanced polymorphism. Lastly, the alleles occur within regulatory sequences, and result in differential sensitivity to environmental perturbation. Together the data provide insight into how genetic, epigenetic and evolutionary forces interact to shape the social brain.

Keywords: cognitive ecology, balancing selection, enhancer elements, single nucleotide polymorphism, Microtus ochrogaster, neuroendocrinology, monogamy

Individual differences in social behavior are remarkably common. Male lizards vary dramatically in their display colors and aggressive behaviors (Sinervo and Lively, 1996). Male sunfish may differ profoundly in their parental care (Gross, 1991), while bluehead wrasses can shift body color, behavior, and even sex in response to social environments (Semsar and Godwin, 2004). Indeed, evolutionary theory has long known that the fitness value of specific behavioral traits may depend on the frequency of such traits in the population, or on the population density of conspecifics (Maynard-Smith and Price, 1973). Similar forces have been hypothesized to shape individual differences in human personality (Keller and Miller, 2006; Penke et al., 2007), resilience to developmental trauma (Boyce and Ellis, 2005), and even the variety of human faces (Sheehan and Nachman, 2014). Understanding the genetic and epigenetic factors that shape individual differences in social behavior is thus of fundamental importance to both our basic understanding of behavior, and to our understanding of natural variation related to health and disease.

Behavioral neuroscience is often focused on model species in which genetic diversity has been intentionally purged. This has the advantage of minimizing variation that could confound 
the study of species-specific traits, and this strategy has enabled substantial insights into the role of developmental factors in shaping adult behavior. Intrauterine environments (Ryan and Vandenbergh, 2002), parental care (Weaver et al., 2004), and environmental complexity (van Praag et al., 2000), for example, all have profound influences on the development of brain and behavior. The decision to study genetically similar individuals, however, precludes studying genetic variation or how it interacts with developmental environments to shape natural behavior.

Non-traditional model species offer a variety of strengths that complement traditional foci of behavioral neuroscience (Phelps, 2010; Taborsky et al., 2015). For example, by studying species in which genetic diversity has been actively retained by derivation from wild stock, it is possible to examine how genetic variation contributes to brain and behavior. In addition, species may be chosen that exhibit interesting social phenotypes not exhibited by traditional model systems. Among mammals, recent examples include the study of pair-bonds (Young and Wang, 2004; Ophir et al., 2007), non-sexual bonds (Beery and Zucker, 2010), elaborate vocalizations (Blondel and Phelps, 2009; Crino et al., 2010), and the elaboration of paternal care (Bendesky et al., 2017). Work on non-traditional rodents and primates, moreover, can employ many of the technologies developed for common mammalian models (e.g., Lim et al., 2004). These attributes make them powerful supplements to common approaches in social neuroscience.

In the current paper, we offer a detailed review of our work on individual differences in the vasopressin system of prairie voles, a socially monogamous rodent that has become a powerful model for the study of attachment. We focus more specifically on cortical differences in the abundance in the vasopressin 1a receptor, the predominant form in the brain. Our focus, the retrosplenial cortex (RSC), is a brain region critical to spatial and contextual memory, and an increasing focus of research in both humans and rodents (Harker and Whishaw, 2002; Vann et al., 2009; Kingsbury et al., 2012; Ranganath and Ritchey, 2012; Cowansage et al., 2014; Todd and Bucci, 2015). The expression of $\mathrm{V} 1 \mathrm{aR}$ in the RSC is profoundly variable among individual prairie voles, and has been linked to both spatial behavior and sexual fidelity in the wild (Figure 1). We begin by introducing prairie voles as models in social neuroscience and neuroendocrinology.

\section{PRAIRIE VOLES AS MODELS OF MONOGAMY}

The prairie vole, Microtus ochrogaster, is a small North American rodent that lives in grasslands. It is known both for its wild fluctuations in population density and for its ability to form enduring pair-bonds (Thomas and Birney, 1979; Carter et al., 1986, 1995; Getz et al., 1993, 2001; Pizzuto and Getz, 1998). Males and females live in pairs and share care of offspring. Roughly $25 \%$ of these young are sired outside the pair (Ophir et al., 2008b). Male pair-bonding is accompanied by a dramatic increase in aggression and a reduction in homerange. While paired males live as aggressive, territorial "residents," up to $45 \%$ of males may
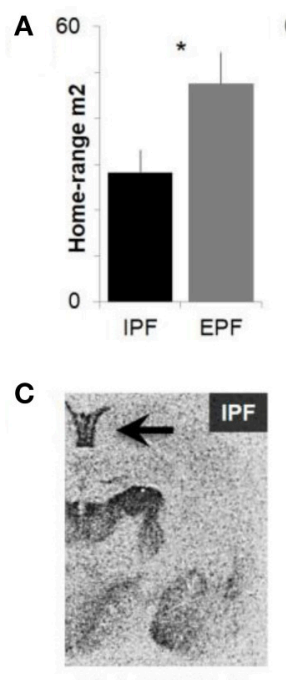

High RSC-V1aR
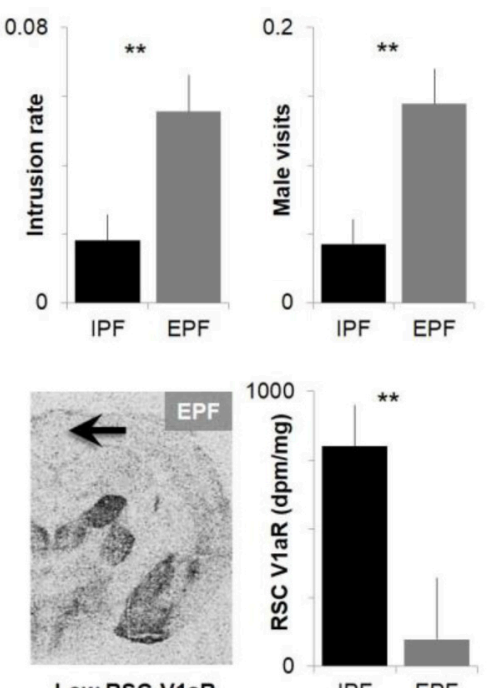

Low RSC-V1aR

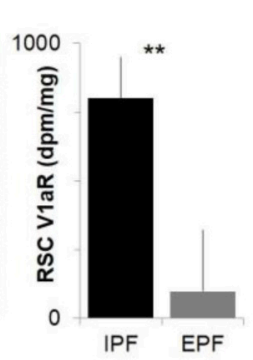

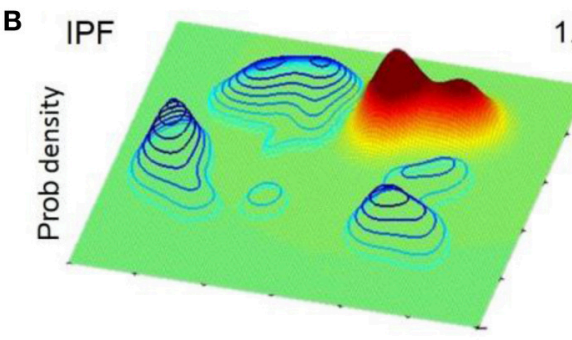

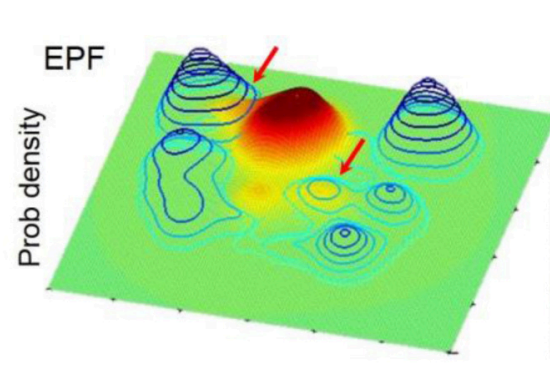

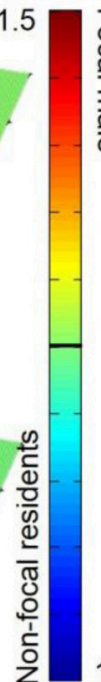

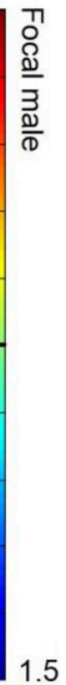

FIGURE 1 | Individual differences in male space-use and sexual fidelity are predicted by RSC-V1aR abundance. (A) Males who sired young only within a pair (intrapair fertilization, IPF) and males who sired at least one embryo with a non-partner (extrapair fertilization, EPF) differ in homerange size, the rate of intrusions onto resident male territories, and the rate at which their own core homeranges were visits by other males. (B) Sample probability density estimates of paired males within a common enclosure. Focal males are shown on a green-red scale and a solid surface, non-focal residents in blue wired surface. $X$ and $Y$ axes correspond to dimensions of the outdoor enclosure. Top panel shows an IPF male that does not intrude on the territories of other bonded males; bottom panel depicts an EPF male who intrudes on two other male territories. Colorbar depicts probability density $(0-1.5 \times 10$ - 3$)$ that a focal male or non-focal resident will be at a given point in space. (C) IPF and EPF males differ in the abundance of $\mathrm{V} 1 \mathrm{aR}$ in the RSC, which predicts individual differences in space use. ${ }^{\star} P<0.05$, ${ }^{\star \star} P<0.01$. Modified with permission from Okhovat et al. (2015). 
live as unpaired, non-territorial "wanderers" (Getz et al., 1993; Solomon and Jacquot, 2002).

Space use is a critical component of variation both within and between these alternative male tactics. Residents with small, exclusive homeranges have high mating success with their respective partners (Ophir et al., 2008c; Phelps and Ophir, 2009). Residents with larger homeranges gain extra-pair fertilizations (EPFs) but are more often cuckolded. Wanderers have larger, less exclusive homeranges than residents, but only those with the largest homeranges obtain EPFs (Ophir et al., 2008c; Phelps and Ophir, 2009). Thus, for both residents and wanderers, larger and less exclusive homeranges translate into increases in extrapair paternity; only residents, however, face trade-offs between EPFs and IPFs. Space use differs between residents and wanderers, but it also predicts patterns of paternity within tactics.

\section{VASOPRESSIN AND MATING SYTEM}

Arginine-vasopressin (AVP) is a nine-amino acid peptide implicated in a wide variety of social behaviors. Among vertebrates, AVP and its homologs are commonly linked to aggression, courtship and other social behaviors (Goodson and Bass, 2001; Caldwell et al., 2008). Among mammals, neurons of the bed nucleus of the stria terminalis and the medial amygdala express AVP at higher levels in males than females (De Vries et al., 1994), a finding thought to contribute to the importance of the peptide to male social behavior (Cho et al., 1999; but see Bosch, 2013; Dumais and Veenema, 2016, for examples of vasopressin functions in female social behaviors). Although this neuropeptide is consistently implicated in social behavior, its effects can be highly species-specific. This specificity seems to emerge from species differences in the neural distribution of the vasopressin 1a receptor (V1aR). Prairie voles, for example, exhibit high $\mathrm{V} 1 \mathrm{aR}$ in a reward region, the ventral pallidum, that influences pairbond formation (Winslow et al., 1993; Insel et al., 1994). Injection of a vasopressin antagonist into the ventral pallidum blocks pair-bonding in male prairie voles (Lim and Young, 2004). Remarkably, viral overexpression of pallidal V1aR enables normally promiscuous male meadow voles to form attachments (Lim et al., 2004).

Although the ventral pallidum causes species differences in pair-bond formation among voles, this mechanism does not seem to be general. We recently measured pallidal V1aR in seven species of Peromyscus, for example, and found it did not reliably predict mating system across deer mice (Turner et al., 2010). The consensus seems to be that the ability of vasopressin and its homologs to modulate social behavior is an ancient innovation common among vertebrates (Goodson, 2005; Ho et al., 2010; O'Connell and Hofmann, 2011, 2012). The effects of the hormone on a particular social behavior, however, can emerge in a variety of ways, presumably by acting anywhere in a series of connected brain regions important for social behavior (e.g., Goodson, 2005; O'Connell and Hofmann, 2011, 2012).

Although differences between monogamous and promiscuous vole species are shaped by pallidal V1aR, residents and wanderers have identical patterns of neural V1aR (Ophir et al., 2008c).
The abundance of V1aR in the ventral pallidum is remarkably consistent across individual prairie voles, with the high levels needed for pair-bonding apparently fixed within the population (Phelps and Young, 2003). Given that bonded males have higher fitness, it seems likely that selection has cleared heritable variation in pallidal V1aR abundance (Phelps and Ophir, 2009). Differences between resident and wandering males seem to represent differences in opportunity rather than neural V1aR abundance (Ophir et al., 2008c). Somewhat surprisingly, although there are no differences in V1aR between residents and wanderers, more subtle behavioral variation within each tactic is associated with the abundance of V1aR in the RSC (Ophir et al., 2008c; Figure 1).

To examine this relationship, we collared and radiotracked animals in the field, using the locations determined over the course of a few weeks to estimate the probability a given animal would be at a particular point in space (Ophir et al., 2008c; Okhovat et al., 2015; Figure 1). From these probability landscapes, we can estimate the core of an animal's homerange, and the extent to which the animal intrudes into the core homeranges of its neighbors. The data reveal that having low RSC-V1aR is associated with more territorial intrusion, increased rates of being intruded upon, and increased extra-pair paternity (Phelps and Ophir, 2009; Okhovat et al., 2015; Figure 1). [Interestingly, RSC-V1aR was not associated with female behavior (Zheng et al., 2013)]. Together these data suggest that vasopressin function shapes individual differences in memory, space-use and sexual fidelity in the field. Given the prominent role of the RSC in spatial memory, we hypothesize that males with low RSC-V1aR are less adept at remembering the spatial location of social interactions. In this scenario, low RSC-V1aR males intrude more because they are less able to recall the details of a punitive encounter with a resident male; males with high RSC-VlaR, in contrast, seem to avoid intruding on male territories, and so are better equipped to guard their mates. An alternative (but not mutually exclusive) hypothesis is that RSC-VlaR influences space use and sexual fidelity by shaping the strength of a bond, or by promoting a male's ability to keep track of his mate. These alternatives have yet to be tested.

\section{GENETIC VARIATION AT THE AVPR1A LOCUS PREDICTS RSC-V1AR EXPRESSION}

Having identified profound individual differences in cortical V1aR (Phelps and Young, 2003), and linked them to individual differences in behavior (Ophir et al., 2008c; Phelps and Ophir, 2009), we next asked whether individual differences in RSCV1aR abundance were genetic, epigenetic, or both. From our field data, there were two plausible explanations: that differences in behavioral experiences somehow drove the individual differences in V1aR, or that V1aR in the RSC preceded and perhaps caused the behavioral differences in space-use and fidelity. If RSC-V1aR 
variation caused behavioral differences, what was the origin of the cortical variation?

A variety of findings suggested that RSC-V1aR was not caused by the experience of intra-pair or extra-pair paternity, but was some complex combination of genetic and developmental regulation of the avpr1a locus. First, there are no sex differences in RSC-V1aR abundance, nor are there any differences between paired and single animals (Phelps and Young, 2003; Ophir et al., 2008c), suggesting that neither sex steroids nor mating experience influenced expression. Moreover, work by Hammock and Young (2005) bred lines of prairie voles with long or short microsatellite lengths in the avprla promoter, and found that they differed substantially in RSC-V1aR abundance. This demonstrates that cis-acting sequence variation contributes to RSC-V1aR. The story became more complicated, however, because neither Hammock et al. (2005), nor our own lab (Ophir et al., 2008a) found microsatellite length to predict
RSC-V1aR in outbred animals. Our hypothesis was that the avprla microsatellite is not causal, but that it was imperfectly linked to neighboring single-nucleotide polymorphisms (SNPs) that are responsible for individual differences. Studies that bred for long or short microsatellites would also select for different frequencies of any linked SNPs.

To test the hypothesis that SNPs were shaping RSC-V1aR abundance, we looked at natural variation in RSC-V1aR and sequence variants from a large population of lab-reared and wild-caught prairie voles across $\sim 8 \mathrm{~kb}$ of the avpr1a locus (Figure 2). We found 151 SNPs overall (Okhovat et al., 2015). None of these SNPs predicted V1aR in brain regions implicated in bonding and aggression (ventral pallidum or lateral septum). However, we found four tightly linked SNPs predicted RSCV1aR. These four SNPs were found upstream of the first exon (SNP -1392), within the intron (SNP 2170 and 2676) and in the second exon (SNP 3506; all SNPs are numbered with
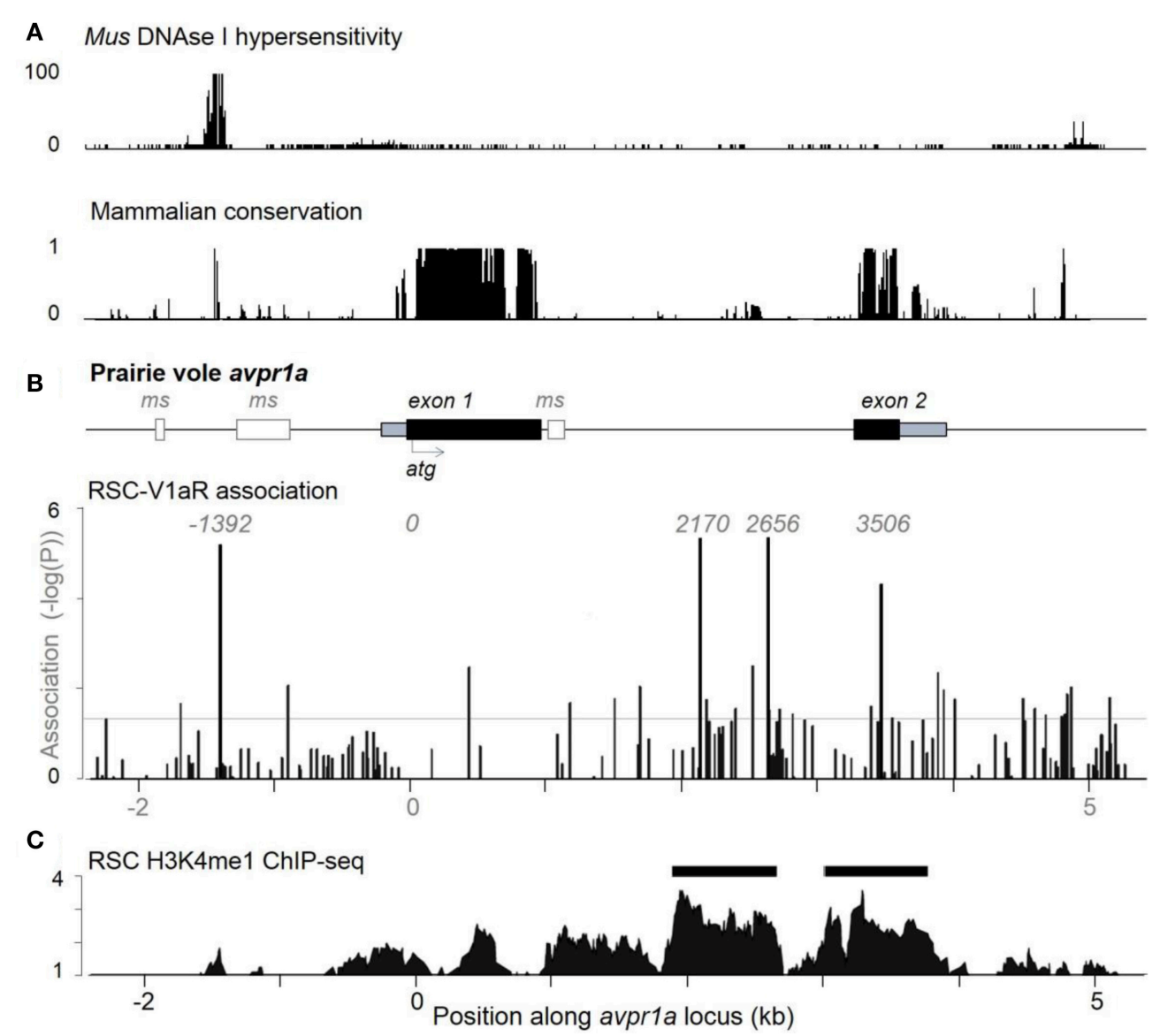

FIGURE 2 | Individual differences in RSC-V1aR abundance are well predicted by 4 linked single nucleotide polymorphisms at the avpr1a locus. (A) ENCODE data on DNAse hypersensivity (top) from the cortex of adult Mus musculus (top), and conservation of corresponding sequences across mammals (below). (B) The structure of the prairie vole avpr1a locus includes two exons (UTRs in gray, CDS in black), and three microsatellite sequences (ms, white). The microsatellite upstream of the first exon has been the subject of numerous studies. Below, vertical bars represent the strength of association (-logP) between each identified SNP and RSC-V1aR abundance. Four strongly linked SNPs (positions -1392, 2170, 2656, 3506) were highly associated with RSC-V1aR and survived multiple comparison corrections. Horizontal bar corresponds to uncorrected $\alpha(P=0.05)$. (C) Chromatin immunoprecipitation sequencing (ChIP-seq) targeting the enhancer marker H3K4me1 reveals significant enrichment (compared to input DNA controls) within the intron, as well as within the second exon. Horizontal scale depicts position in kilobases (kb) of avpr1a locus, aligned to all panels in figure. Data used in association analyses included both males and females. Modified with permission from Okhovat et al. (2015). 
respect to translation start site; Figure $2 \mathrm{~B}$ ). We refer to the set of SNPs that correspond to high RSC-V1aR as the HI allele, and the opposite set of SNPs as the LO allele. We replicated this association on a third population of wild-derived animals, crossing parents heterozygous for the alleles to produce $\mathrm{HI} / \mathrm{HI}$ and LO/LO homozygotes in the same litter (Okhovat et al., 2015). We found that the HI and LO alleles were strong, replicable, and robust predictors of not only RSC-V1aR, but also avpr1a transcript abundance, suggesting that these predictive SNPs affect avpr1a cis-regulation.

When located within regulatory regions, SNPs can alter gene expression by changing the epigenetic properties of the locus. Remarkably, all four RSC-associated avpr1a SNPs co-localized with markers of gene regulation. SNP -1392 was within an deoxyribonuclease I (DNAse I) hypersensitive site, a marker of open chromatin; moreover, this open chromatin was centered on a highly conserved binding site for the transcription factor CTCF, a factor known to shape gene regulation through its contributions to chromatin looping (Phillips and Corces, 2009, Figure 2A). Distal regulatory sequences that interact directly with promoters to regulate transcription are known as enhancers, and can be identified through their characteristic histone modifications (Heintzman et al., 2009). One such mark is the monomethylation of lysine 4 in histone 3 (H3K4me1), which marks both active and poised enhancers (Heintzman et al., 2009). We performed ChIPseq on the RSC of prairie voles, and found that one such enhancer site was located in the avprla intron and overlapped with both SNP 2170 and SNP 2676 (Figure 2C). There was also a second putative enhancer that overlapped with the second avprla exon and SNP 3506 in the HI/LO alleles.

Interestingly, SNP 2170 is a T/G polymorphism that alters the presence/absence of a $\mathrm{CpG}$ site located within a putative intron enhancer. This site is also weakly linked to additional polymorphic $\mathrm{CpG}$ sites (polyCpG) within the same enhancer, leading to significant $\mathrm{HI}$ and $\mathrm{LO}$ allelic differences in $\mathrm{CpG}$ availability; the LO allele, which is associated with lower RSC$\mathrm{V} 1 \mathrm{aR}$, has significantly more $\mathrm{CpG}$ sites in the putative intron enhancer compared to the HI allele (Okhovat et al., 2015, 2017b). CpG sites are the main targets for DNA methylationa well-known epigenetic modification that can regulate gene expression-therefore, we hypothesized that avprla genotype differences in enhancer $\mathrm{CpG}$ could lead to differences in enhancer methylation and avprla expression in the RSC.

We found that both lab-reared and wild-caught showed significantly different levels of DNA methylation in the intron enhancer (Okhovat et al., 2015, 2017b). There was also a negative correlation between overall enhancer methylation and avprla transcription (Okhovat et al., 2015, 2017a), suggesting that enhancer methylation lowers RSC-V1aR by reducing avpr1a transcription, consistent with commonly reported silencing effects of DNA methylation (Nan et al., 1998). While enhancer methylation predicted individual differences in RSC-V1aR, methylation of the avpria promoter did not (Okhovat et al., 2017a). Although promoter methylation is generally silencing (Bird and Wolffe, 1999), our data indicate that the avpr1a promoter is generally un-methylated, whether the locus is active or not. This finding is in line with recent studies that suggest promoters are often unmethylated, even in cell types in which they are not expressed-thus a lack of methylation is necessary but not sufficient for gene expression (Rollins et al., 2006; Lister et al., 2013). Methylation and sequence variation in regulatory elements outside of the promoter areaespecially within enhancer sequences-may be better predictors of expression.

A detailed analysis of $\mathrm{HI}$ and LO allele sequences suggested at least two mechanisms by which sequence variation and epigenetic mechanisms might interact at the avprla enhancer. First, allelic differences in CpG abundance and overall enhancer methylation could account for differences in expression via allelebiased recruitment of repressive methyl-binding proteins-such as MeCP2 (Bird, 2002). Alternatively, binding of transcription factors may be influenced by sequence changes generated by SNP 2170. Based on published position weight matrices, some transcription factors, including GATA2-which is expressed in the mouse RSC-bind preferably to the LO allele (Okhovat et al., 2017b). Therefore, differential binding of transcription factors due to both genetic and epigenetic variation at the intron enhancer may drive allele-biased changes in RSC-V1aR abundance. While further research is required to elucidate the exact molecular consequences of sequence variation in the intron enhancer, these findings provide promising explanations for the variation observed in RSC-V1aR.

\section{SELECTION MAINTAINS ALLELIC VARIATION RELATED TO RSC-V1AR ABUNDANCE}

Although individuals can vary tremendously in social behavior, as well as in gene expression and brain function, we know relatively little about how DNA sequence variation contributes to meaningful differences in brain and behavior. We have reviewed data showing that individual differences in RSC-V1aR predict behavior of male prairie voles in the field, and that these brain differences are due at least in part to genetic variation at the avprla locus. Here, we examine whether there is evidence that natural selection has actively maintained variation in brain in behavior.

Our first analysis was to revisit data on paternity and fitness obtained from animals in the field (Figure 1). We asked whether there was a difference between paired and single males in their overall fitness, as measured by the number of pups that they sired in the field. We found that paired males sired more young (Ophir et al., 2008b), demonstrating that selection favors the capacity to form pair-bonds. However, we did not find a difference in the fitness of males who mated exclusively with a partner (IPF), and those who mated at least once outside a pairbond (EPF), suggesting that both faithful and unfaithful males do comparably well in the conditions we examined (Ophir et al., 2008c). We examined the relative fitness of $\mathrm{HI}$ and LO RSC alleles in our field study and found that they did not differ significantly in fitness (Figure 3A; Okhovat et al., 2015). However, when we examined how this fitness was obtained, we found that $\mathrm{HI}$ alleles were more fit than LO alleles in the 


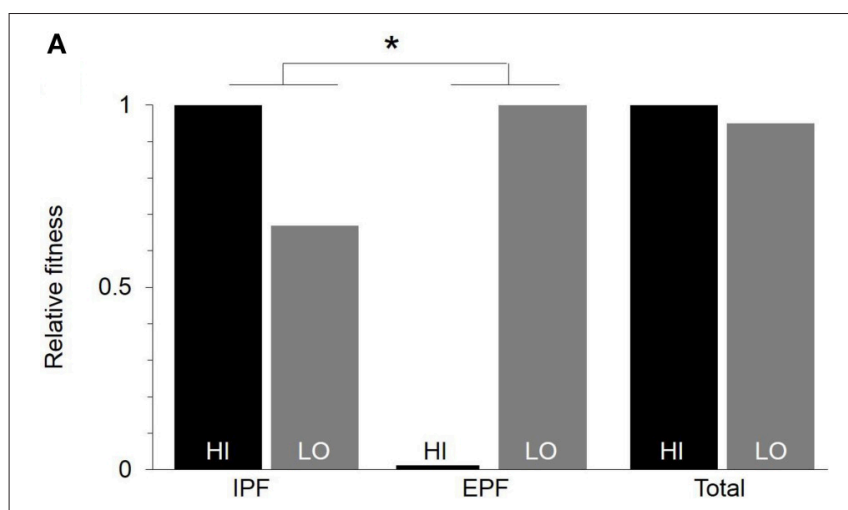

$$
\text { B }
$$

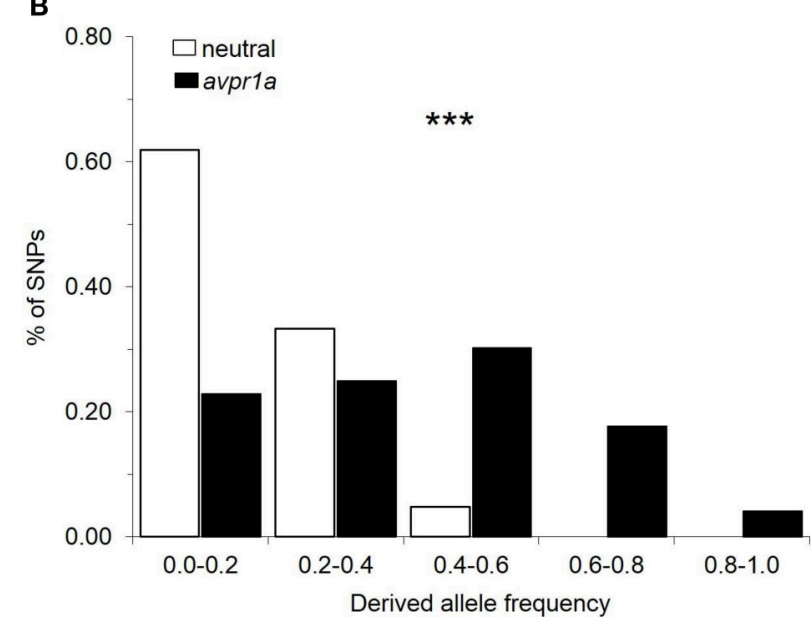

FIGURE 3 | Natural selection maintains variation in RSC-V1aR abundance. (A) Relative fitness of $\mathrm{HI}$ and $\mathrm{LO}$ alleles measured in the context of intrapair (IPF) and extrapair (EPF) fertilization rates obtained by male prairie voles. Selection is measured by the difference in fitness of the two alleles. The differences in the direction and strength of selection in IPF and EPF contexts were tested with a permutation test. (B) Comparison of the frequency spectra of polymorphisms for avpr1a (black) and neutral loci (white) reveals a significant excess of intermediate frequency alleles in avpr1a. ${ }^{*} P<0.05,{ }^{\star \star \star} P<0.001$. Modified with permission from Okhovat et al. (2015). context of intra-pair fertilizations, while LO alleles were more fit in the context of extra-pair fertilizations (Figure 3A; Okhovat et al., 2015). This is consistent with the view that the diversity of V1aR in the RSC represents a "balanced polymorphism" of the social brain, in which faithful and unfaithful male mating behaviors provide alternate but equivalent sources of evolutionary fitness.

While these results were encouraging, our field studies were a snapshot in time, tested under a single set of population densities and over a narrow range of conditions. We used evolutionary genetic approaches to test whether there was a history of selection actively maintaining variation at the avpria locus. A new mutation is, by definition, at low frequency within a population, and in the absence of selection, it is more likely to be lost than to drift to high frequency; thus most variable sites in the genome are at low frequencies (Hudson et al., 1987; Tajima, 1989). If selection is actively maintaining alternative forms of an allele, however, both forms tend to be at intermediate frequencies, and neighboring sites are also at higher frequencies than is characteristic of the genome as a whole (Hudson et al., 1987; Tajima, 1989). We compared the frequencies of mutations at the avpria locus to those in three putatively neutral genes (Okhovat et al., 2015), or across the entire genome (Berrío Escobar, 2017). We found that indeed, the avprla locus had higher frequencies of SNPs than was characteristic of the rest of the genome, suggesting that selection actively maintained this diversity (Figure 3B). Moreover, this signal was concentrated in the vicinity of the SNPs that defined the HI and LO allelesa region of the avprla locus that did not predict expression in other brain regions (Okhovat et al., 2015; Berrío Escobar, 2017). Together these data suggest that RSC-V1aR diversity represents adaptive variation in brain, behavior, and cognition.

The high degree of linkage between the SNPs that defined $\mathrm{HI}$ and LO alleles seems unusual, because many intervening polymorphisms are unlinked to $\mathrm{HI}$ and LO alleles. We used permutation tests to ask whether these SNPs were significantly more linked than we would expect by chance (Berrío Escobar, 2017). We found that the SNPs were significantly more linked than predicted based on the distance between them-a pattern suggesting that the selection had favored specific combination of nucleotides across sites. Such epistasis across regulatory regions is poorly studied, but not without precedent. For example, recent data suggest that SNP-by-SNP interactions among noncoding elements play an important role in human disease (Dinu et al., 2012; Jamshidi et al., 2015). Such epistasis may reflect interactions among transcription factors that bind at different sites, contributions to chromatin looping and conformation, or any of the many other molecular changes needed to effectively coordinate transcription at a locus (e.g., Grubert et al., 2015). Whether the HI and LO SNPs interact remains to be determined, but our evidence of non-random linkage further suggests a causal role for these polymorphisms.

\section{DEVELOPMENTAL VARIATION AT THE AVPR1A LOCUS}

Although SNPs in avpr1a regulatory sequences seem to have a major role in regulating RSC-V1aR abundance, a variety of data suggested that environmental factors may also be at play. For example, lab-reared voles had a stronger association between $\mathrm{HI}$ and LO alleles and RSC-V1aR abundance than did wildcaught prairie voles (Okhovat et al., 2015). This observation suggested that RSC-V1aR variation might also be shaped by the environmental variation that voles are naturally exposed to in the wild (e.g., population and resource fluctuations, Getz et al., 2001). In fact, previous work on prairie voles (Bales et al., 2007; Prounis et al., 2015) and rats (Francis et al., 2002) demonstrated that developmental manipulations can alter $\mathrm{V} 1 \mathrm{aR}$ regulation in the $\mathrm{RSC}$ and other brain regions. While the exact molecular mechanisms for these neuronal changes are not known, environmentally induced changes in neuronal gene expression are often mediated by molecular epigenetic modifications, such as DNA methylation (Szyf and Bick, 2013). 
Given that $\mathrm{HI}$ and $\mathrm{LO}$ alleles differ in the abundance of $\mathrm{CpG}$ sites within the putative intron enhancer, and that the methylation of this enhancer is negatively associated with RSC-V1aR abundance

(Okhovat et al., 2015, Figure 4A), we hypothesized that LO

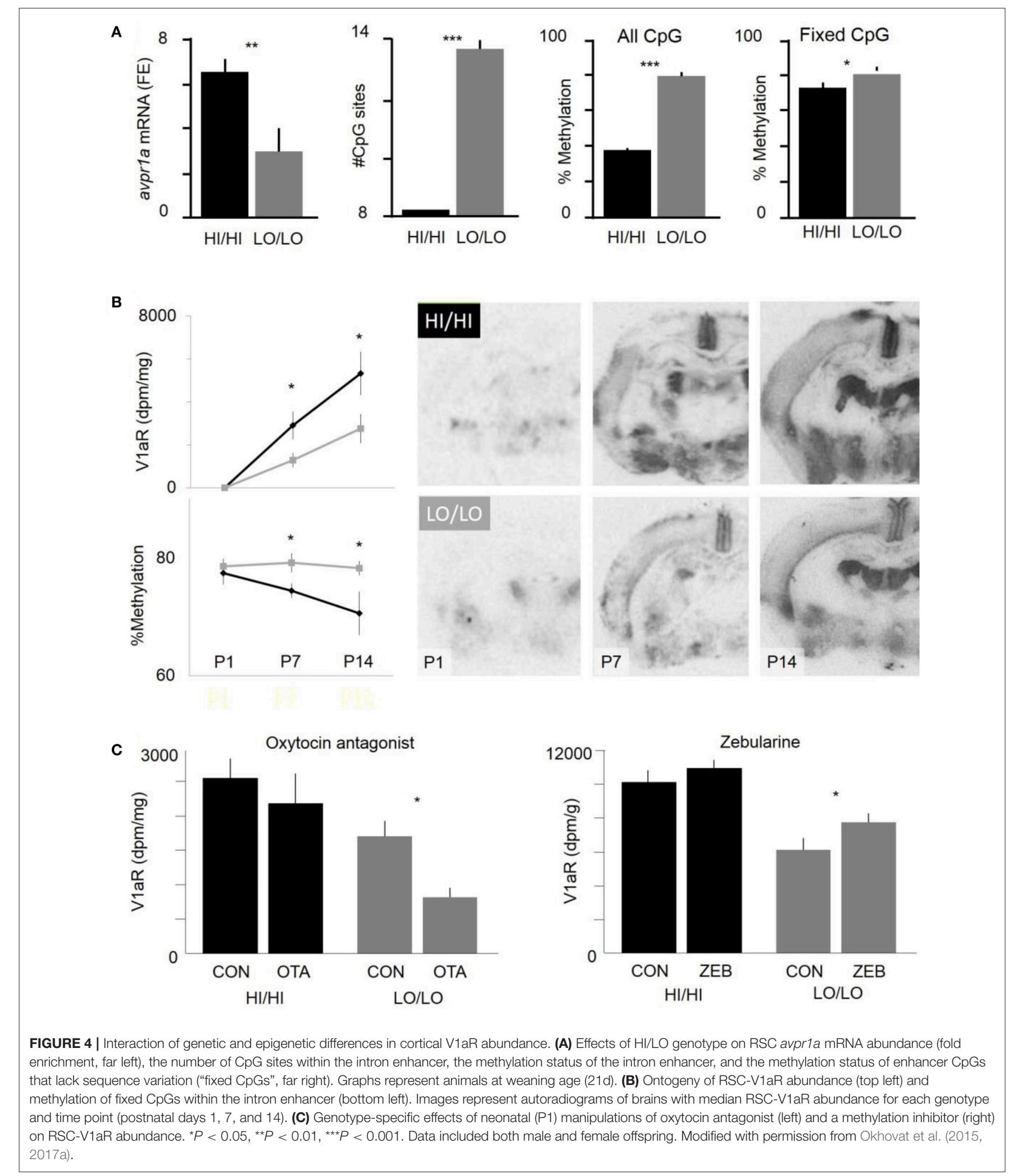


alleles may be more sensitive to developmental perturbations that influence CpG methylation.

Typically, rodent brains undergo periods of dramatic developmental change in gene expression and methylation; such critical periods are often highly responsive to environmental variation in parental care, diet, or stress (Roth and Sweatt, 2011). However, based on genetic makeup, individuals can vary in their sensitivity and response to these early developmental perturbations, a phenomenon known as gene-by-environment interactions $(\mathrm{GxE})$. In prairie voles, neuronal V1aR abundance undergoes drastic changes postnatally (Wang et al., 1997). To begin to understand how genotype interacts with development, we examined the ontogeny of RSC-V1aR in $\mathrm{HI} / \mathrm{HI}$ and $\mathrm{LO} / \mathrm{LO}$ genotypes. We found that one-day-old $\mathrm{HI} / \mathrm{HI}$ and $\mathrm{LO} / \mathrm{LO}$ voles lacked RSC-V1aR (Okhovat et al., 2017a). However, significant genotype differences in RSC-V1aR quickly emerge during the first postnatal week (Figure 4B). Interestingly, genotype differences in avprla enhancer methylation also appear during this period, indicating that enhancer methylation may be involved in early-life regulation of RSC-V1aR (Okhovat et al., 2017a, Figure 4B).

To assess HI and LO differences in susceptibility to earlylife perturbation, newborn pups were exposed to oxytocin receptor antagonist, a manipulation that is sometimes considered analogous to poor parenting, and that has been shown to alter adult RSC-V1aR of voles (Bales et al., 2007). This postnatal treatment reduced RSC-V1aR later at weaning age (21 days), demonstrating that avpr1a regulation is sensitive to early developmental and environmental perturbations (Okhovat et al., 2017a, Figure 4C). This sensitivity, however, was only detected in LO/LO pups, and not their HI/HI siblings. Similarly, we used a global inhibitor of methylation, zebularine (Cheng et al., 2003) to manipulation methylation in newborn pups. We found that zebularine treatment increased RSC-V1aR in LO/LO 21d animals but not in their HI/HI siblings (Okhovat et al., 2017a, Figure 4C). Overall, these data present a remarkably coherent picture in which the high CpG density of LO alleles made them both more sensitive to the silencing effects of the oxytocin receptor antagonist, and to the demethylating effects of zebularine. LO alleles seem to be more developmentally sensitive, while HI alleles seem to be constitutively highly expressing.

While HI and LO alleles differ in their sensitivity to developmental perturbation, examination of the methylation of the intron enhancer suggests a more complex story than we hypothesized. Enhancer methylation was not influenced by these developmental manipulations (Okhovat et al., 2017a). While HI and LO genotypes exhibited GxE interactions, this effect does not seem to be due to CpG density differences in the putative intron enhancer alone. It is likely that genetic differences in the intron enhancer are inherited along with genetic variation at additional unexamined enhancers. Indeed, methyl-DNA immunoprecipitation (meDIP) identifies additional differentially methylated near avpr1a, but outside of our original focus (Okhovat et al., 2017a, Figure 5). Examining whether any of these sites also contain sequence differences between HI and LO alleles may clarify how genetic variation in avprla regulatory mechanisms contributes to sensitivity to developmental perturbation, and how these interact with regulatory regions we have already identified.

\section{CONCLUSIONS AND FUTURE DIRECTIONS}

Our work began with the observation that the distribution of vasopressin 1a receptor in the RSC was surprisingly variable across individuals (Insel et al., 1994; Phelps and Young, 2003). We found that this variation predicted patterns of spaceuse and sexual fidelity in the field, with high levels of RSC$\mathrm{V} 1 \mathrm{aR}$ associated with sexual fidelity, and low levels associated with infidelity-even among paired males (Ophir et al., 2008c; Okhovat et al., 2015). Field paternity data and patterns of standing variation within the genome both suggest that variation at the avprla locus has been actively maintained by selection. Lastly, the alleles that drive differences in RSC-V1aR influence not only the mean level of vasopressin receptor, but also its sensitivity to developmental perturbation. Although this work spans diverse levels of analysis, from the function of chromatin to tests of selection in natural environments, there are a number of interesting questions that remain unanswered.

From a molecular perspective, while $\mathrm{HI}$ and $\mathrm{LO}$ alleles cause differences in RSC-V1aR abundance, we do not yet understand how nucleotide variation translates into differences in avpr1a function. Which of the four linked SNPs, if any, are causal? The case is strongest for the intron SNP 2170: it is a polymorphic CpG site associated with a cluster of polymorphic CpGs; it occurs within a region of chromatin that displays an enhancerspecific histone mark; it exhibits differential methylation between genotypes; its methylation status is associated with RSC-V1aR in animals from both lab and field; and it exhibits a pattern of nucleotide diversity that indicates a history of balancing selection (Okhovat et al., 2015, 2017a,b; Berrío Escobar, 2017). The 5' SNP $(-1396)$ has been less studied, but is also promising. It flanks a strongly conserved CTCF binding site and resides within a region of open chromatin (Okhovat et al., 2015, Figure 1A). The unusually tight linkage between these sites similarly suggests some coordinated function (Berrío Escobar, 2017). These data, however, fall short of demonstrating that either of these SNPs is causal. Moreover, the fact that developmental perturbations influence RSC-V1aR without altering the methylation status of the intron enhancer suggest that there are other, more distal regulators-an interpretation reinforced by the existence of differentially methylated regions outside of the immediate avpr1a locus (Figure 5). Whether such distal regulators bear sequence variation that contributes to $\mathrm{HI}$ and $\mathrm{LO}$ alleles remains to be determined. Our ChIP-seq approach allows for the exhaustive identification of distal regulatory sites, but conformation capture methods such as $\mathrm{Hi}-\mathrm{C}$ will be needed to identify sites that make contact with avprla promoter, and that are thus likely to be directly shaping avpr1a function (Mifsud et al., 2015). Gene therapy methods using cas9 to target deletions of putative enhancers, or using inactivated cas 9 fused to chromatin-remodeling enzymes to shape the function of specific regulatory sequences (Senís et al., 2014) 


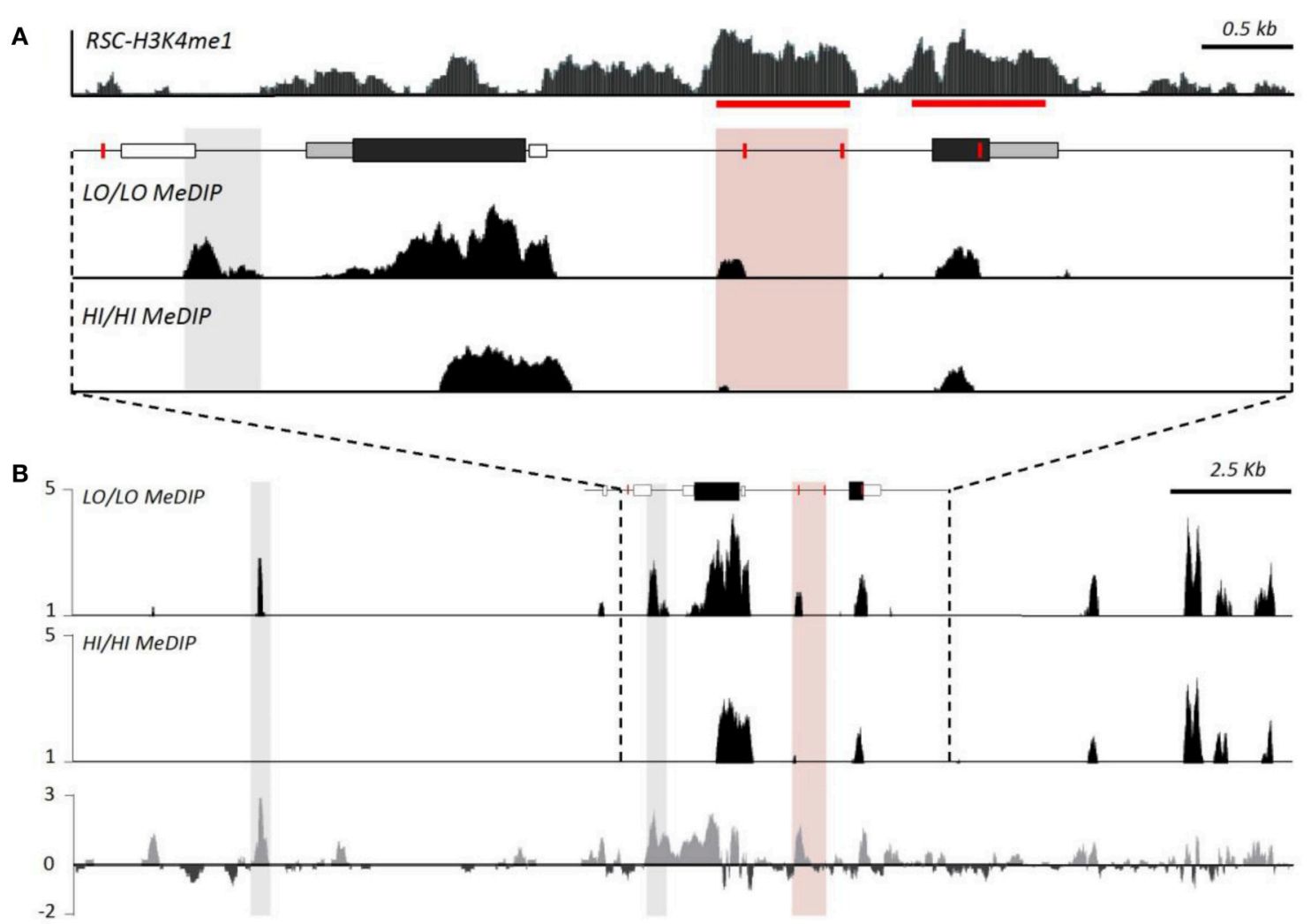

FIGURE 5 | Genotype differences in methylation suggest distal regulators of avpr1a function. (A) Top panel depicts relative read depth (fold enrichment) of H3K4me1 reads denoting putative enhancers at the avpr1a locus. Bottom panels depict read depths from methyl-DNA immunoprecipitation sequencing (meDIP) targeting the $\mathrm{RSC}$ of $\mathrm{HI} / \mathrm{HI}$ and LO/LO prairie voles. The data confirm enhanced methylation near SNP 2170 (pink box) of LO/LO genotypes, as well as revealed a differentially methylated region (DMR) just upstream of the transcription start site (where there are no genetic differences between $\mathrm{HI}$ and LO alleles). (B) A more expanded view of the locus reveals a strong DMR $10 \mathrm{~kb}$ upstream of the locus; it is not known whether this or other more distal sites also differ in their underlying sequence. Modified with permission from Okhovat et al. (2017b).

provide a means for more directly determining whether specific nucleotides shape cortical expression of the avpria locus, and how such nucleotides interact with developmental experience.

While the molecular underpinnings of RSC-V1aR will offer novel insights into the nature of GxE and their substrates, a second series of unanswered questions concerns the exact nature of the relationship between RSC-V1aR and behavior. The behavioral functions of the RSC are an area of active investigation in both humans and traditional laboratory rodents. From a neuroanatomical perspective, the RSC is interconnected with the hippocampus, entorhinal cortex, anterior thalamus, and laterodorsal thalamus - a circuit central to episodic and spatial memory (Aggleton, 2014). Indeed, the RSC is active during navigation tasks, and in rats the RSC contains head-direction cells (Vann et al., 2009; Todd and Bucci, 2015). Imaging studies of humans (and rodents) at rest reveal that the RSC is one of two major nodes of the "default mode network" - a group of brain regions active when not performing a task (Spreng et al., 2008; Lu et al., 2012; Stafford et al., 2014). The second major node is the anterior cingulate cortex, a major target of the RSC (Spreng et al.,
2008; Lu et al., 2012; Stafford et al., 2014). One interpretation is that the RSC connects a posterior circuit that processes memory, with a more anterior prefrontal circuit that processes decisionmaking; in human studies, the default mode network activity is sometimes interpreted as daydreaming, in which memory is used to simulate possible actions (Spreng et al., 2008).

Causal manipulations of RSC function confirm its role in a variety of memory-related tasks, but there is not a clear consensus on exactly how the RSC contributes to memory. In one recent study, Cowansage et al. (2014) used activity-dependent expression of channel rhodospins to tag and manipulate RSC neurons that were active during exposure to a shockassociated context. They found that activation of these neurons could elicit freezing responses in the absence of the context. One interpretation of these data is that the RSC serves to either encode or retrieve long-term memories and, through its reciprocal projections with the hippocampus, allow access to those memories during related experiences (Todd and Bucci, 2015).

The existing literature suggests a variety of alternative hypotheses for the role of RSC-V1aR in space-use and sexual 
fidelity. Our core observation is that a male with high V1aR intrudes less on territories of neighboring males, more effectively guards his mate, and mates predominantly with his partner. One hypothesis is that animals with high $\mathrm{VlaR}$ are better able remember the locations of social interactions-this could translate into the observed patterns of space-use and fidelity by making high $\mathrm{V} 1 \mathrm{aR}$ males better able to guard mates (Okhovat et al., 2015). Similarly, having low cortical V1aR may impair the ability to recall locations of punitive encounters, making low-V1aR males more likely to intrude on neighboring territories and gain extra-pair copulations (Ophir et al., 2008c). In addition, there may be something non-spatial about the role of the RSC in social interaction-it may shape memory for one's partner, for example, or facilitate discrimination between remembered individuals through its projections to prefrontal cortices. Whatever the pattern proves to be, a rich set of studies aimed at dissecting the cognitive aspects of bonding, navigation, choice, and fidelity remain to be done.

Aside from the specific insights the above studies offer, they also provide a general framework for thinking about variation in the nervous system and its relationship to social behavior. First, they demonstrate that genetic variation in brain function can be a source of adaptive behavioral variation within a species. Our understanding of genetic variation in the nervous system is incredibly understudied, and this work provides a novel perspective on how diverse brains can be. A second value is that the studies illustrate how modern tools

\section{REFERENCES}

Aggleton, J. P. (2014). Looking beyond the hippocampus: old and new neurological targets for understanding memory disorders. Proc. R. Soc. B 281:20140565. doi: 10.1098/rspb.2014.0565

Bales, K. L., van Westerhuyzen, J. A., Lewis-Reese, A. D., Grotte, N. D., Lanter, J. A., and Carter, C. S. (2007). Oxytocin has dose-dependent developmental effects on pair-bonding and alloparental care in female prairie voles. Horm. Behav. 52, 274-279. doi: 10.1016/j.yhbeh.2007.05.004

Beery, A. K., and Zucker, I. (2010). Oxytocin and same-sex social behavior in female meadow voles. Neuroscience 169, 665-673. doi: 10.1016/j.neuroscience.2010.05.023

Bendesky, A., Kwon, Y.-M., Lassance, J.-M., Lewarch, C. L., Yao, S., Peterson, B. K., et al. (2017). The genetic basis of parental care evolution in monogamous mice. Nature 544, 434-439. doi: 10.1038/nature22074

Berrío Escobar, A. (2017). Gene Regulatory Evolution and the Origin of Complex Behaviors in the Prairie Vole, Microtus ochrogaster. Thesis. Available online at: https://repositories.lib.utexas.edu/handle/2152/46829

Bird, A. (2002). DNA methylation patterns and epigenetic memory. Genes Dev. 16, 6-21. doi: 10.1101/gad.947102

Bird, A. P., and Wolffe, A. P. (1999). Methylation-induced repressionBelts, braces, and chromatin. Cell 99, 451-454. doi: 10.1016/S0092-8674(00) 81532-9

Blondel, D. V., and Phelps, S. M. (2009). Space use and social structure of long-tailed singing mice (Scotinomys xerampelinus). J. Mammal. 90, 715-723. doi: 10.1644/08-MAMM-A-009R2.1

Bosch, O. J. (2013). Maternal aggression in rodents: brain oxytocin and vasopressin mediate pup defence. Phil. Trans. R. Soc. B 368:20130085. doi: 10.1098/rstb.2013.0085

Boyce, W. T., and Ellis, B. J. (2005). Biological sensitivity to context: I. an evolutionary-developmental theory of the origins and functions of for interrogating chromatin function can be used to identify specific DNA sequences likely to be important to the regulation of behavior. On a genome scale, combining these sequencing tools with evolutionary genetics will allow researchers to more quickly identify which among the many thousands of regulatory sequences (and billions of nucleotides) are likely to be playing a causal role in gene expression (e.g., Pollard et al., 2006; Boyd et al., 2015). Lastly, we show how identifying nucleotide variation within specific regulatory sequences allows one to explore the interactions between genetic and epigenetic variation. Together such approaches will be a tremendous aid not only to our understanding of natural behavior, but in our quest to identify how variation in the genome interacts with the environment to shape the diversity of social behavior related to both health and disease.

\section{AUTHOR CONTRIBUTIONS}

SP conceived and outlined the review, wrote the introduction, conclusion and future directions, assigned manuscript tasks and revised all other sections. MO wrote the sections on genetics and epigenetics. $\mathrm{AB}$ wrote the section on evolutionary analyses.

\section{FUNDING}

This work was funded by NSF IOS-1457350 and 1355188 awarded to SP. stress reactivity. Dev. Psychopathol. 17, 271-301. doi: 10.1017/S09545794050 50145

Boyd, J. L., Skove, S. L., Rouanet, J. P., Pilaz, L.-J., Bepler, T., Gordân, R., et al. (2015). Human-chimpanzee differences in a fzd8 enhancer alter cell-cycle dynamics in the developing neocortex. Curr. Biol. 25, 772-779. doi: 10.1016/j.cub.2015.01.041

Caldwell, H. K., Lee, H.-J., Macbeth, A. H., and Young, W. S. (2008). Vasopressin: behavioral roles of an original neuropeptide. Prog. Neurobiol. 84, 1-24. doi: 10.1016/j.pneurobio.2007.10.007

Carter, C. S., Devries, A. C., and Getz, L. L. (1995). Physiological substrates of mammalian monogamy: the prairie vole model. Neurosci. Biobehav. Rev. 19, 303-314. doi: 10.1016/0149-7634(94)00070-H

Carter, C. S., Getz, L. L., and Cohen-Parsons, M. (1986). Relationships between social organization and behavioral endocrinology in a monogamous mammal. Adv. Study Behav. 16, 109-145. doi: 10.1016/S0065-3454(08)60189-8

Cheng, J. C., Matsen, C. B., Gonzales, F. A., Ye, W., Greer, S., Marquez, V. E., et al. (2003). Inhibition of DNA methylation and reactivation of silenced genes by zebularine. J. Natl. Cancer Inst. 95, 399-409. doi: 10.1093/jnci/95.5.399

Cho, M. M., Courtney, A., Williams, J. R., and Sue, C. (1999). The effects of oxytocin and vasopressin on partner preferences in male and female prairie voles (Microtus ochrogaster). Behav. Neurosci. 113, 1071-1079. doi: $10.1037 / 0735-7044.113 .5 .1071$

Cowansage, K. K., Shuman, T., Dillingham, B. C., Chang, A., Golshani, P., and Mayford, M. (2014). Direct reactivation of a coherent neocortical memory of context. Neuron 84, 432-441. doi: 10.1016/j.neuron.2014.09.022

Crino, O. L., Larkin, I., and Phelps, S. M. (2010). Stress coping styles and singing behavior in the short-tailed singing mouse (Scotinomys teguina). Horm. Behav. 58, 334-340. doi: 10.1016/j.yhbeh.2010.02.011

De Vries, G., Wang, Z., Bullock, N. A., and Numan, S. (1994). Sex differences in the effects of testosterone and its metabolites on vasopressin messenger RNA levels in the bed nucleus of the stria terminalis of rats. J. Neurosci. 14, 1789-1794. 
Dinu, I., Mahasirimongkol, S., Liu, Q., Yanai, H., Eldin, N. S., Kreiter, E., et al. (2012). SNP-SNP interactions discovered by logic regression explain Crohn's disease genetics. PLoS ONE 7:e43035. doi: 10.1371/journal.pone.0043035

Dumais, K. M., and Veenema, A. H. (2016). Vasopressin and oxytocin receptor systems in the brain: sex differences and sex-specific regulation of social behavior. Front. Neuroendocrinol. 40:03. doi: 10.1016/j.yfrne.2015.04.003

Francis, D. D., Young, L. J., Meaney, M. J., and Insel, T. R. (2002). Naturally occurring differences in maternal care are associated with the expression of oxytocin and vasopressin (V1a) receptors: gender differences. J. Neuroendocrinol. 14, 349-353. doi: 10.1046/j.0007-1331.2002.00776.x

Getz, L. L., Hofmann, J. E., McGuire, B., and Dolan, T. W. III (2001). Twenty-five years of population fluctuations of Microtus ochrogaster and $M$. pennsylvanicus in three habitats in east-Central Illinois. J. Mammal. 82, 22-34. doi: 10.1644/1545-1542(2001)082\&lt;0022:TFYOPF\&gt;2.0.CO;2

Getz, L. L., McGuire, B., Pizzuto, T., Hofmann, J. E., and Frase, B. (1993). Social Organization of the prairie vole (Microtus ochrogaster). J. Mammal. 74, 44-58. doi: $10.2307 / 1381904$

Goodson, J. L. (2005). The vertebrate social behavior network: evolutionary themes and variations. Horm. Behav. 48, 11-22. doi: 10.1016/j.yhbeh.2005.02.003

Goodson, J. L., and Bass, A. H. (2001). Social behavior functions and related anatomical characteristics of vasotocin/vasopressin systems in vertebrates. Brain Res. Rev. 35, 246-265. doi: 10.1016/S0165-0173(01)00043-1

Gross, M. R. (1991). Evolution of alternative reproductive strategies: frequencydependent sexual selection in male bluegill sunfish. Philos. Trans. Biological Sci. 332, 59-66. doi: 10.1098/rstb.1991.0033

Grubert, F., Zaugg, J. B., Kasowski, M., Ursu, O., Spacek, D. V., Martin, A. R., et al. (2015). Genetic control of chromatin states in humans involves local and distal chromosomal interactions. Cell 162, 1051-1065. doi: 10.1016/j.cell.2015.07.048

Hammock, E. A. D., and Young, L. J. (2005). Microsatellite instability generates diversity in brain and sociobehavioral traits. Science 308, 1630-1634. doi: 10.1126/science.1111427

Hammock, E. A. D., Lim, M. M., Nair, H. P., and Young, L. J. (2005). Association of vasopressin 1a receptor levels with a regulatory microsatellite and behavior. Genes Brain Behav. 4, 289-301. doi: 10.1111/j.1601-183X.2005. 00119.x

Harker, K. T., and Whishaw, I. Q. (2002). Impaired spatial performance in rats with retrosplenial lesions: importance of the spatial problem and the rat strain in identifying lesion effects in a swimming pool. J. Neurosci. 22, 1155-1164. Available online at: http://www.jneurosci.org/content/22/3/1155. long

Heintzman, N. D., Hon, G. C., Hawkins, R. D., Kheradpour, P., Stark, A., Harp, L. F., et al. (2009). Histone modifications at human enhancers reflect global celltype-specific gene expression. Nature 459, 108-112. doi: 10.1038/nature07829

Ho, J. M., Murray, J. H., Demas, G. E., and Goodson, J. L. (2010). Vasopressin cell groups exhibit strongly divergent responses to copulation and male-male interactions in mice. Horm. Behav. 58, 368-377. doi: 10.1016/j.yhbeh.2010.03.021

Hudson, R. R., Kreitman, M., and Aguadé, M. (1987). A test of neutral molecular evolution based on nucleotide data. Genetics 116, 153-159.

Insel, T., Wang, Z., and Ferris, C. (1994). Patterns of brain vasopressin receptor distribution associated with social-organization in microtine rodents. J. Neurosci. 14, 5381-5392.

Jamshidi, M., Fagerholm, R., Khan, S., Aittomäki, K., Czene, K., Darabi, H., et al. (2015). SNP-SNP interaction analysis of NF- $\kappa B$ signaling pathway on breast cancer survival. Oncotarget 6, 37979-37994. doi: 10.18632/oncotarget.4991

Keller, M. C., and Miller, G. (2006). Resolving the paradox of common, harmful, heritable mental disorders: which evolutionary genetic models work best?. Behav. Brain Sci. 29, 385-404.

Kingsbury, M. A., Gleason, E. D., Ophir, A. G., Phelps, S. M., Young, L. J., and Marler, C. A. (2012). Monogamous and promiscuous rodent species exhibit discrete variation in the size of the medial prefrontal cortex. Brain Behav. Evol. 80, 4-14. doi: 10.1159/000339247

Lim, M. M., Wang, Z., Olazábal, D. E., Ren, X., Terwilliger, E. F., and Young, L. J. (2004). Enhanced partner preference in a promiscuous species by manipulating the expression of a single gene. Nature 429, 754-757. doi: 10.1038/nature02539

Lim, M. M., and Young, L. J. (2004). Vasopressin-dependent neural circuits underlying pair bond formation in the monogamous prairie vole. Neuroscience 125, 35-45. doi: 10.1016/j.neuroscience.2003.12.008
Lister, R., Mukamel, E. A., Nery, J. R., Urich, M., Puddifoot, C. A., Johnson, N. D., et al. (2013). Global epigenomic reconfiguration during mammalian brain development. Science 341:1237905. doi: 10.1126/science.1237905

Lu, H., Zou, Q., Gu, H., Raichle, M. E., Stein, E. A., and Yang, Y. (2012). Rat brains also have a default mode network. Proc. Natl. Acad. Sci. U.S.A. 109, 3979-3984. doi: 10.1073/pnas.1200506109

Maynard-Smith, J., and Price, G. R. (1973). The logic of animal conflict. Nature 246, 15-18. doi: 10.1038/246015a0

Mifsud, B., Tavares-Cadete, F., Young, A. N., Sugar, R., Schoenfelder, S., Ferreira, L., et al. (2015). Mapping long-range promoter contacts in human cells with high-resolution capture Hi-C. Nat. Genet. 47, 598-606. doi: 10.1038/ ng.3286

Nan, X., Ng, H.-H., Johnson, C. A., and Laherty, C. D. (1998). Transcriptional repression by the methyl-CpG-binding protein $\mathrm{MeCP} 2$ involves a histone deacetylase complex. Nature 393, 386-389. doi: 10.1038/30764

O'Connell, L. A., and Hofmann, H. A. (2011). The vertebrate mesolimbic reward system and social behavior network: a comparative synthesis. J. Comp. Neurol. 519, 3599-3639. doi: 10.1002/cne.22735

O'Connell, L. A., and Hofmann, H. A. (2012). Evolution of a vertebrate social decision-making network. Science 336, 1154-1157. doi: 10.1126/science. 1218889

Okhovat, M., Berrio, A., Wallace, G., Ophir, A. G., and Phelps, S. M. (2015). Sexual fidelity trade-offs promote regulatory variation in the prairie vole brain. Science 350, 1371-1374. doi: 10.1126/science.aac5791

Okhovat, M., Chen, I. C., Dehghani, Z., Zheng, D. J., Ikpatt, J. E., Momoh, H., et al. (2017a). Genetic variation in the developmental regulation of cortical avprla among prairie voles. Genes Brain Behav. doi: 10.1111/gbb.12396. [Epub ahead of print].

Okhovat, M., Maguire, S. M., and Phelps, S. M. (2017b). Methylation of avpr1a in the cortex of wild prairie voles: effects of $\mathrm{CpG}$ position and polymorphism. Open Sci. 4:160646. doi: 10.1098/rsos.160646

Ophir, A. G., Campbell, P., Hanna, K., and Phelps, S. M. (2008a). Field tests of cis-regulatory variation at the prairie vole avprla locus: association with V1aR abundance but not sexual or social fidelity. Horm. Behav. 54, 694-702. doi: 10.1016/j.yhbeh.2008.07.009

Ophir, A. G., Phelps, S. M., Sorin, A. B., and Wolff, J. O. (2007). Morphological, genetic, and behavioral comparisons of two prairie vole populations in the field and laboratory. J. Mammal. 88, 989-999. doi: 10.1644/06-MAMMA-250R.1

Ophir, A. G., Phelps, S. M., Sorin, A. B., and Wolff, J. O. (2008b). Social but not genetic monogamy is associated with greater breeding success in prairie voles. Anim. Behav. 75, 1143-1154. doi: 10.1016/j.anbehav.2007.09.022

Ophir, A. G., Wolff, J. O., and Phelps, S. M. (2008c). Variation in neural VlaR predicts sexual fidelity and space use among male prairie voles in semi-natural settings. Proc. Natl. Acad. Sci. U.S.A. 105, 1249-1254. doi: 10.1073/pnas.0709116105

Penke, L., Denissen, J. J. A., and Miller, G. F. (2007). The evolutionary genetics of personality. Eur. J. Pers. 21, 549-587. doi: 10.1002/per.629

Phelps, S. M. (2010). From endophenotypes to evolution: social attachment, sexual fidelity and the avprla locus. Curr. Opin. Neurobiol. 20, 795-802. doi: 10.1016/j.conb.2010.09.002

Phelps, S. M., and Ophir, A. G. (2009). "Monogamous brains and alternative tactics: neuronal V1aR, space use, and sexual infidelity among male prairie voles," in Cognitive Ecology II, eds R. Dukas and J. M. Ratcliffe (University of Chicago Press), 156-176. doi: 10.7208/chicago/9780226169378. 003.0009

Phelps, S. M., and Young, L. J. (2003). Extraordinary diversity in vasopressin (V1a) receptor distributions among wild prairie voles (Microtus ochrogaster): patterns of variation and covariation. J. Comp. Neurol. 466, 564-576. doi: 10.1002/cne.10902

Phillips, J. E., and Corces, V. G. (2009). CTCF: master weaver of the genome. Cell 137, 1194-1211. doi: 10.1016/j.cell.2009.06.001

Pizzuto, T., and Getz, L. L. (1998). Female prairie voles (Microtus ochrogaster) fail to form a new pair after loss of mate. Behav. Processes 43, 79-86. doi: 10.1016/S0376-6357(97)00091-0

Pollard, K. S., Salama, S. R., Lambert, N., Lambot, M.-A., Coppens, S., Pedersen, J. S., et al. (2006). An RNA gene expressed during cortical development evolved rapidly in humans. Nature 443, 167-172. doi: 10.1038/nature05113 
Prounis, G. S., Foley, L., Rehman, A., and Ophir, A. G. (2015). Perinatal and juvenile social environments interact to shape cognitive behaviour and neural phenotype in prairie voles. Proc. R. Soc. Lond. B Biol. Sci. 282:20152236. doi: $10.1098 / \mathrm{rspb} .2015 .2236$

Ranganath, C., and Ritchey, M. (2012). Two cortical systems for memory-guided behaviour. Nat. Rev. Neurosci. 13, 713-726. doi: 10.1038/nrn3338

Rollins, R. A., Haghighi, F., Edwards, J. R., Das, R., Zhang, M. Q., Ju, J., et al. (2006). Large-scale structure of genomic methylation patterns. Genome Res. 16, 157-163. doi: 10.1101/gr.4362006

Roth, T. L., and Sweatt, J. D. (2011). Epigenetic mechanisms and environmental shaping of the brain during sensitive periods of development. J. Child Psychol. Psychiatry 52, 398-408. doi: 10.1111/j.1469-7610.2010.02282.x

Ryan, B. C., and Vandenbergh, J. G. (2002). Intrauterine position effects. Neurosci. Biobehav. Rev. 26, 665-678. doi: 10.1016/S0149-7634(02)00038-6

Semsar, K., and Godwin, J. (2004). Multiple mechanisms of phenotype development in the bluehead wrasse. Horm. Behav. 45, 345-353. doi: 10.1016/j.yhbeh.2004.01.003

Senís, E., Fatouros, C., Große, S., Wiedtke, E., Niopek, D., Mueller, A.-K., et al. (2014). CRISPR/Cas9-mediated genome engineering: an adeno-associated viral (AAV) vector toolbox. Biotechnol. J. 9, 1402-1412. doi: 10.1002/biot.201400046

Sheehan, M. J., and Nachman, M. W. (2014). Morphological and population genomic evidence that human faces have evolved to signal individual identity. Nat. Commun. 5:4800. doi: 10.1038/ncomms5800

Sinervo, B., and Lively, C. M. (1996). The rock-paper-scissors game and the evolution of alternative male strategies. Nature 380, 240-243. doi: $10.1038 / 380240 \mathrm{a} 0$

Solomon, N. G., and Jacquot, J. J. (2002). Characteristics of resident and wandering prairie voles, Microtus ochrogaster. Can. J. Zool. 80, 951-955. doi: 10.1139/z02-053

Spreng, R. N., Mar, R. A., and Kim, A. S. N. (2008). The common neural basis of autobiographical memory, prospection, navigation, theory of mind, and the default mode: a quantitative meta-analysis. J. Cogn. Neurosci. 21, 489-510. doi: 10.1162/jocn.2008.21029

Stafford, J. M., Jarrett, B. R., Miranda-Dominguez, O., Mills, B. D., Cain, N., Mihalas, S., et al. (2014). Large-scale topology and the default mode network in the mouse connectome. Proc. Natl. Acad. Sci. U.S.A. 111, 18745-18750. doi: $10.1073 /$ pnas. 1404346111

Szyf, M., and Bick, J. (2013). DNA methylation: a mechanism for embedding early life experiences in the genome. Child Dev. 84, 49-57. doi: 10.1111/j.1467-8624.2012.01793.x

Taborsky, M., Hofmann, H. A., Beery, A. K., Blumstein, D. T., Hayes, L. D., Lacey, E. A., et al. (2015). Taxon matters: promoting integrative studies of social behavior. Trends Neurosci. 38, 189-191. doi: 10.1016/j.tins.2015.01.004
Tajima, F. (1989). Statistical method for testing the neutral mutation hypothesis by DNA polymorphism. Genetics 123, 585-595.

Thomas, J. A., and Birney, E. C. (1979). Parental care and mating system of the prairie vole, Microtus ochrogaster. Behav. Ecol. Sociobiol. 5, 171-186. doi: 10.1007/BF00293304

Todd, T. P., and Bucci, D. J. (2015). Retrosplenial cortex and long-term memory: molecules to behavior. Neural Plast. 2015:414173. doi: 10.1155/2015/414173

Turner, L. M., Young, A. R., Römpler, H., Schöneberg, T., Phelps, S. M., and Hoekstra, H. E. (2010). Monogamy evolves through multiple mechanisms: evidence from V1aR in deer mice. Mol. Biol. Evol. 27, 1269-1278. doi: 10.1093/molbev/msq013

Vann, S. D., Aggleton, J. P., and Maguire, E. A. (2009). What does the retrosplenial cortex do? Nature Rev. Neurosci. 10, 792-802. doi: 10.1038/nrn2733

van Praag, H., Kempermann, G., and Gage, F. H. (2000). Neural consequences of enviromental enrichment. Nat. Rev. Neurosci. 1, 191-198. doi: $10.1038 / 35044558$

Wang, Z., Young, L. J., Liu, Y., and Insel, T. R. (1997). Species differences in vasopressin receptor binding are evident early in development: comparative anatomic studies in prairie and montane voles. J. Comp. Neurol. 378, 535-546.

Weaver, I. C. G., Cervoni, N., Champagne, F. A., D’Alessio, A. C., Sharma, S., Seckl, J. R., et al. (2004). Epigenetic programming by maternal behavior. Nat. Neurosci. 7, 847-854. doi: 10.1038/nn1276

Winslow, J. T., Hastings, N., Carter, C. S., Harbaugh, C. R., and Insel, T. R. (1993). A role for central vasopressin in pair bonding in monogamous prairie voles. Nature 365, 545-548. doi: 10.1038/365545a0

Young, L. J., and Wang, Z. (2004). The neurobiology of pair bonding. Nat. Neurosci. 7, 1048-1054. doi: 10.1038/nn1327

Zheng, D. J., Larsson, B., Phelps, S. M., and Ophir, A. G. (2013). Female alternative mating tactics, reproductive success and nonapeptide receptor expression in the social decision-making network. Behav. Brain Res. 246, 139-147. doi: 10.1016/j.bbr.2013.02.024

Conflict of Interest Statement: The authors declare that the research was conducted in the absence of any commercial or financial relationships that could be construed as a potential conflict of interest.

Copyright (c) 2017 Phelps, Okhovat and Berrio. This is an open-access article distributed under the terms of the Creative Commons Attribution License (CC BY). The use, distribution or reproduction in other forums is permitted, provided the original author(s) or licensor are credited and that the original publication in this journal is cited, in accordance with accepted academic practice. No use, distribution or reproduction is permitted which does not comply with these terms. 